\title{
La fibra de coco como sustituto de la turba en la obtención de plántulas de uchuva (Physalis peruviana L.)
}

\section{Coco peat as a substitute for peat moss in the production of cape gooseberry (Physalis peruviana L.) seedlings}
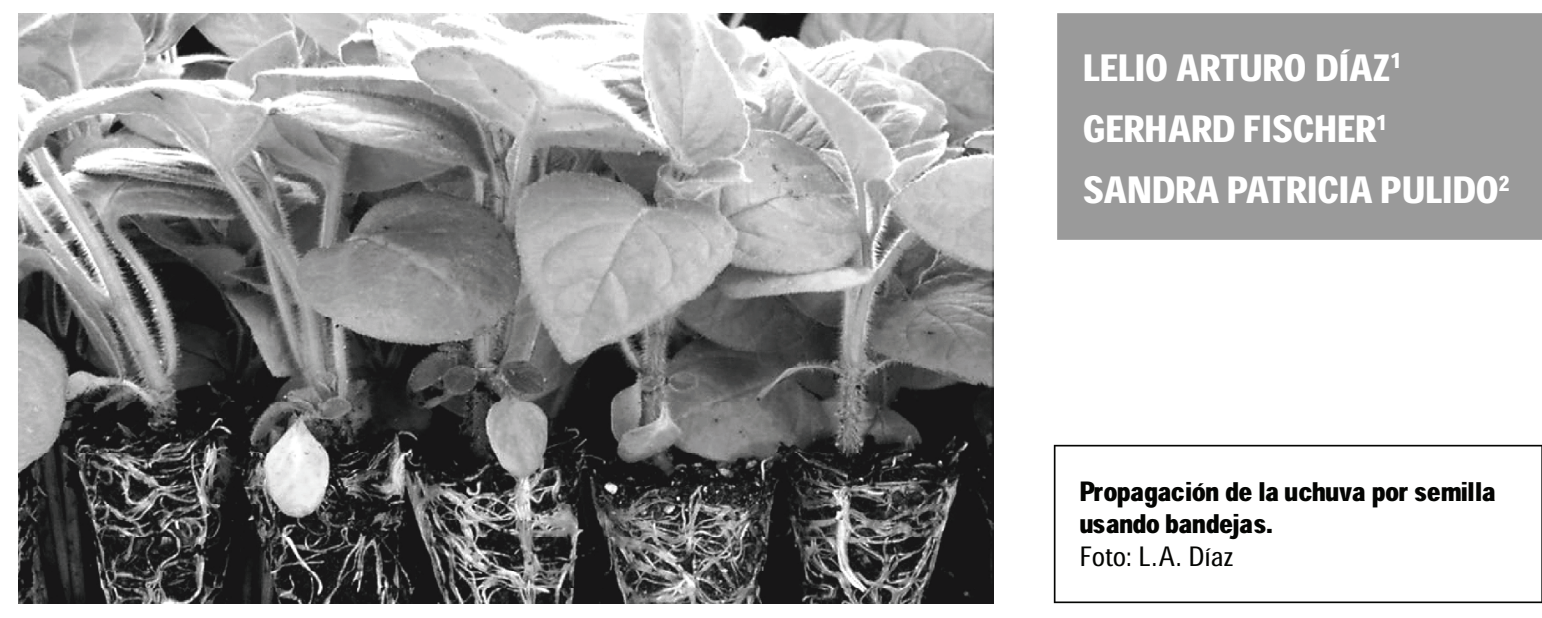

\section{RESUMEN}

Se evaluó la emergencia de semillas de uchuva con dos tamaños diferentes para obtener plántulas cultivadas bajo invernadero en diferentes sustratos en bandejas alveoladas, en condiciones de manejo comercial. Los dos tamaños de la semilla se obtuvieron mediante tamizado, a partir de frutos clasificados por sanidad y peso: 1,107 y 0,916 mg, que resultaron en un $100 \%$ de germinación en laboratorio. En invernadero se sembraron las uchuvas en seis sustratos: fibra de coco brasilera (FCB), arena lavada de río fina (ALR), turba al emana rubia (TAR), turba canadiense con perlita (TCP), mezcla de turba alemana rubia con cascarilla de arroz 50:50 (TAR+C) y una mezcla de fibra de coco brasilera con cascarilla de arroz 50:50 (FCB +C). Se evaluó emergencia, altura, número y área de hojas, masa seca de la parte aérea y volumen de raíces de las plántulas en invernadero. La FCB presentó los valores más altos para altura y número de hojas, mientras este sustrato y la TAR indujeron la mayor área foliar y masa seca de la plántula. El volumen de raíz fue promovido en todos los sustratos del estudio que contuvieron turba, y también en la FCB. En la ALR se registraron los resultados más bajos para todas las variables, especialmente en el desarrollo radical. Las semillas más grandes $(1,107 \mathrm{mg})$ produjeron la mayor biomasa seca de la parte aérea de la plántula.

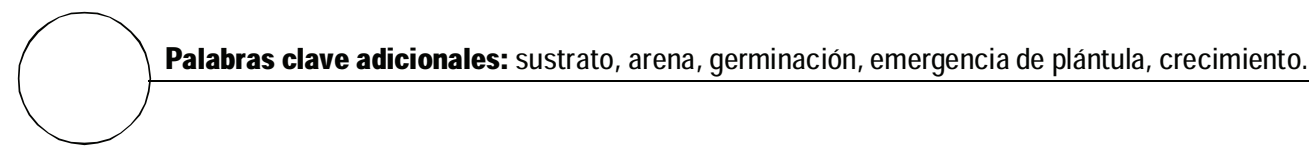

\footnotetext{
Facultad de Agronomía, Departamento de Agronomía, Universidad Nacional de Colombia, Bogotá (Colombia)

2 Centro de Investigaciones y Asesorías Agroindustriales (CIAA; ahora: Centro de Bio-sistemas), Universidad Jorge Tadeo Lozano, Chía (Colombia)

3 Autor para correspondencia.gfischer@unal.edu.co
} 


\section{ABSTRACT}

The emergence of two seed sizes of the cape gooseberry for obtaining seedlings was evaluated. The plants were cultivated in a greenhouse using different substrates with egg flats, under commercial production conditions. The two seed sizes were sieved from fruits classified by health and weight: 1.107 and $0.916 \mathrm{mg}$, which had $100 \%$ germination in the laboratory. In the greenhouse, the cape gooseberry seedlings were planted in six substrates: Brazilian coco peat (BCP), fine river sand (FRS), German blond peat moss (GBP), Canadian peat moss with perlite (CPP), a mix of German blond peat moss with rice hulls 50:50 (GBP+R) and a mix of coco peat and rice hulls 50:50 (BCP+R). This study evaluated emergence, height, leaf area and number, dry matter of the aerial plant part and root volume of the seedlings in the greenhouse. The BCP substrate produced the highest values for seedling height and leaf number; and also, along with GBP, induced the highest leaf area and plant dry matter. Root volume was promoted in all substrates which contained peat moss and in BCP. In FRS, the lowest values in all variables were measured, especially in root development. The biggest seeds (1.107 $\mathrm{mg}$ ) produced the majority of their dry biomass from the aerial part of the seedling.

Additional key words: substrate, sand, germination, seedling emergency, growth.

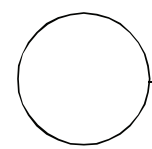

El género Physalis (familia Solanaceae) comprende entre 75 y 90 especies (Whitson y Manos, 2005), cuyos frutos se forman y permanecen dentro del cáliz durante todo su desarrollo (Legge, 1974), y pertenecen al tipo de las bayas, debido a que el endocarpio y mesocarpio son carnosos (Ryugo, 1993) y a que contienen numerosas semillas, entre 100 y 300. Su forma es globosa u ovoide, con un diámetro entre 1,25 y $2,50 \mathrm{~cm}$ y pesa de 4 a $10 \mathrm{~g}$; se desarrolla y madura durante 60 a $80 \mathrm{~d}$, dependiendo de las condiciones agroecológicas de la plantación (Fischer, 2000).

Las semillas de uchuva son pequeñas, de forma lenticular, y están desprovistas de hilos placentarios (Valencia, 1985). En diversos estudios (Almanza, 2000; Peña et al., 2010; Fischer et al., 2007) se ha encontrado que el peso promedio de 1.000 semillas de uchuva es de $1 \mathrm{~g}$. Un fruto de las uchuvas, ecotipo 'Colombia', produce en promedio 210 a $240 \mathrm{mg}$ de semillas, que corresponden a 250 a 320 unidades por fruto (Almanza, 2000; Fischer et al., 2007). Brücher (1977) menciona que con el incremento del tamaño de la semilla de uchuva, usándola en la propagación, los frutos de la siguiente cosecha son más grandes; igualmente, Jansen et al. (1998) reportan que semillas más pesadas pueden producir plantas más vigorosas con rendimientos más altos.

Las semillas de uchuva pasan de un color blanquecino a verde amarillento a los $20 \mathrm{~d}$; siguen a amarillo verdoso a los $49 \mathrm{~d}$, y finalmente a pardo claro, que es cuando el fruto alcanza su madurez fisiológica, y las semillas están aptas para germinar (Almanza y Espinosa, 1995). En las condiciones de Tunja (Boyacá), esta coloración inició a los $42 \mathrm{~d}$ del desarrollo del fruto, para el ecotipo Colombia, presentándose en ese momento un $20 \%$ de germinación, y el $52 \%$ se alcanzó a los 49 d del inicio de la coloración amarillo verdoso, característico porcentaje que aumentó al alcanzar el color pardo claro (Almanza y Espinosa, 1995).

El término de la germinación se define como el proceso de reactivación del metabolismo de la semilla y la emergencia de la radícula y de la plúmula, conducentes a producción de una plántula; y para las pruebas con semillas y propagación de plantas, la definición de la germinación debe incluir la producción de una plántula normal (Hartmann et al., 2005). La emergencia de las semillas depende de características fisiológicas y bioquímicas, de su reacción al ambiente y su eficiencia en el uso de 
sus propias reservas durante la germinación (Celis-Velázquez et al., 2005).

Las semillas poseen reservas que alimentarán la nueva plántula hasta que esta pueda establecerse como un organismo fotosintéticamente competente (autótrofo). Cuando las condiciones del medio son favorables para germinar, inicia la regeneración de la planta madre (Mantilla, 2008). Los elementos minerales depositados en la semillas son removilizados y transportados a los órganos crecientes de la plántula joven (Schopfer y Brennicke, 2006).

Las plantas obtenidas a partir de semillas son más vigorosas, tienen un sistema radical bien desarrollado, fuerte y profundo y son más longevas, en comparación con la propagación asexual (Borscak y Covatta, 2007). Las etapas de obtención, manejo de la semilla y manejo de los semilleros son esenciales para el establecimiento del cultivo en términos fisiológicos y de sanidad (Hartmann et al., 2005).

Sustratos son todos los materiales de origen natural, mineral u orgánico, que colocados en un contenedor, y gracias a sus características físicas y químicas, permiten el anclaje del sistema radicular. La finalidad del uso de sustratos apropiados es garantizar la germinación de la semilla y brindar soporte para plántulas en el más corto lapso (Abad et al., 2004), además de proporcionar la aireación que necesitan las raíces de las plantas para el intercambio de oxígeno y nutrientes; esto dependerá principalmente del tamaño y distribución de los poros para una adecuada retención de agua disponible (Ansorena, 1994).

Debido a la carencia de información sobre los mejores sustratos para la germinación, emergencia y obtención de plántulas de uchuva, se llevó acabo este estudio, con el fin de contribuir con el conocimiento para mejorar los resultados en la propagación sexual de esta especie.

\section{MATERIALES Y MÉTODOS}

El trabajo experimental se realizó en el CIAA (ahora: Centro de Bio-sistemas) de la Universidad Jorge Tadeo Lozano, ubicado en el municipio de
Chía (Cundinamarca) a una altitud de 2.600 msnm. Con el fin de mantener control mayor sobre las variables que inciden en el comportamiento de las plantas y facilitar el mane-jo, las semillas fueron sembradas en bandejas de 128 alvéolos bajo invernadero cubierto en plástico. Este invernadero disponía de un sistema de camas soporte de bandejas de propagación, polisombra y sistema de fertirriego con manguera. La temperatura promedio en el día fue de $22^{\circ} \mathrm{C}$ y en la noche de $18^{\circ} \mathrm{C}$. La solución fertilizante que se utilizó fue la estándar para germinación y obtención de plántulas de $\mathrm{NH}_{4}$ 31-34, $\mathrm{NO}_{3} 117, \mathrm{P}_{2} \mathrm{O}_{5} 54, \mathrm{~K}_{2} \mathrm{O} 154, \mathrm{CaO} 104, \mathrm{Mg}$ 30 y S $22 \mathrm{mg} \mathrm{L}^{-1}$.

La semilla se obtuvo manualmente por presión mecánica y lavado manual y secado de fruta de exportación ecotipo 'Colombia', proveniente de la finca «Los Arrayanes», con certificación Eurepgap, ubicada en la vereda Boquerón Alto, municipio de Chocontá (Cundinamarca), a una altura de $2.650 \mathrm{msnm}$.

Se clasificó la mejor fruta por tamaño, color, sanidad sin rajado, obteniéndose tres diferentes tamaños de fruta, los cuales registraron también diferentes tamaños de semilla y, por consiguiente, pesos diferentes de semilla.

El método para obtener la semilla y clasificarla por peso fue cribarla una vez se secó, usando tamices de prueba estándar para cernir suelo en el laboratorio, los cuales están especificados por Ia American Standard Testing Measurement Specifications. La mayoría de semilla $(69,9 \%)$ de más peso pasó por la malla de calibre $14(1,4 \mathrm{~mm}$ de diámetro), con un peso en promedio de 1,10 $\mathrm{mg}$; la segunda cantidad de semilla que se obtuvo fue del $23,6 \%$, que pasó por la malla $16(1,18 \mathrm{~mm}$ de diámetro), con un pesó en promedio de 0,91 mg. Estos dos pesos están exactamente por debajo y por encima del peso estándar de semillas de uchuva, 1,0 mg (Almanza, 2000), tratándose de semillas con un desarrollo normal y plenamente maduras.

Los 6 sustratos utilizados fueron (1) FCB: fibra de coco lista para uso agrícola en semilleros de germinación, marca Golden Mix ${ }^{\circledR}$ Granulado T 47; (2) ALR: arena fina lavada de río inerte; (3) TAR: turba alemana rubia marca Klasmann ${ }^{\circledR}$ 
KTS 1 fina con partículas de $0-5 \mathrm{~mm}$ de estructura, con adición de abono de $1,0 \mathrm{~L}^{-1}$ de sustrato de microelementos, $46 \%$ de carbono de origen orgánico, materia orgánica total $14-16 \mathrm{~kg} /$ $250 \mathrm{~L}$, contenido de sales de $1.000 \mathrm{mg} \mathrm{L}^{-1}$, proporción de N:P:K de 14:16:18 $1 \mathrm{~kg} \mathrm{~m}^{-3}$; (4) TCP: turba canadiense con perlita marca Fafard ${ }^{\circledR}$ Growing Mix F 15 con contenido de sphagnum de $85 \%$ y de perlita del $15 \%$ de $1-2 \mathrm{~mm}$ de diámetro; (5) TAR+C: una mezcla de turba alemana Klasmann ${ }^{\circledR}$ KTS 1 Fina y cascarilla de arroz sin quemar en proporción 50:50; y (6) $\mathrm{FCB}+\mathrm{C}$ : una mezcla de fibra de coco lista Golden Mix ${ }^{\circledR}$ con cascarilla de arroz sin quemar en proporción 50:50.

Tabla 1. Propiedades físicas de los sustratos fibra de coco brasilera (FCB), turba alemana rubia (TAR), turba canadiense con perlita (TCP), cascarilla de arroz (CAS) y arena lavada de río fina (ALR), comparado con un sustrato óptimo (OPT).

\begin{tabular}{|c|c|c|c|c|c|c|c|c|c|c|}
\hline \multirow{3}{*}{ Sustrato } & \multicolumn{2}{|c|}{ Densidad } & \multirow{3}{*}{$\begin{array}{l}\text { Capacidad } \\
\text { de umedad } \\
\text { (\% Vol.) }\end{array}$} & \multicolumn{2}{|c|}{ Porosidad } & \multirow{3}{*}{$\begin{array}{l}\text { Volumen de } \\
\text { aire (\%) }\end{array}$} & \multirow{3}{*}{ pH } & \multirow{3}{*}{$\begin{array}{l}\text { Capacidad } \\
\text { de retención } \\
\text { de agua(\%) }\end{array}$} & \multirow{3}{*}{$\begin{array}{c}\text { CE } \\
\left(\mathrm{mS} \mathrm{cm} \mathrm{cm}^{-1}\right)\end{array}$} & \multirow{3}{*}{$\begin{array}{c}\text { CIC } \\
\text { (meq/100 g) }\end{array}$} \\
\hline & seca & húmeda & & total & de aire & & & & & \\
\hline & \multicolumn{2}{|c|}{$\left(\mathrm{g} \mathrm{cm}^{-3}\right)$} & & \multicolumn{2}{|c|}{ (\% Vol..) } & & & & & \\
\hline FCB & & 0,92 & 92 & 68 & $15-25$ & 11 & 5,8 & 70 & 1,8 & 11,2 \\
\hline TAR & 0,11 & 0,70 & 59 & 84 & 25 & 18 & $5,5-6,5$ & 80 & 30 & $80-180$ \\
\hline TCP & 0,09 & 0,52 & 43 & 76 & 33 & 22 & 6,5 & 75 & & \\
\hline CAS & 0,10 & 1,23 & 12 & 82 & 69 & 10 & 6,0 & 45 & & \\
\hline ALR & 1,44 & 1,83 & 39 & 45 & 6 & 1 & 6,0 & 10 & 4,0 & $10-40$ \\
\hline OPT & 0,15 & 1,50 & $>30$ & $60-75$ & $10-20$ & $>5$ & $5,0-6,0$ & $>50$ & & \\
\hline
\end{tabular}

$\mathrm{CE}$, conductividad eléctrica; $\mathrm{ClC}$, capacidad de intercambio catiónico. Fuente: Hanan (1998); Arias (1989); Jansen et al. (1998).

Una vez se humedeció el sustrato, se llenaron las bandejas -marcadas para cada tratamiento-, se sembró la semilla correspondiente a una profundidad de $1 \mathrm{~cm}$ y se cubrió con el mismo sustrato. Se cubrió por completo con un tapete negro de fibra artificial tejida y se humedeció con suficiente agua, con el fin de favorecer la germinación y mantener las condiciones de temperatura y humedad constantes; además, para evitar pérdida de semilla o sustrato de cobertura debido al fertirriego con manguera.

El diseño experimental se distribuyó en bloques completos al azar. Los tratamientos fueron dos pesos de semilla $x$ seis sustratos $x$ tres repeticiones, para un total de 36 unidades experimentales. Cada unidad experimental fue una bandeja de 128 alvéolos, sembrando 4.608 semillas.

Se efectuó prueba de germinación en el laboratorio de Fisiología Vegetal de la Facultad de Agronomía, sede Bogotá, en la cámara de germinación marca Seedburo Equipment, a una temperatura constante de $28^{\circ} \mathrm{C}$ y $80 \%$ de humedad relativa. Se dispusieron 600 semillas de los dos diferentes pesos en seis cajas de Petri y sobre papel filtro, y se taparon con agua; 100 semillas de cada peso por cada caja y tres repeticiones, y se efectuó un conteo de las semillas germinadas cada 2 días.

Se realizaron mediciones con 2 días de intervalo a partir de la siembra para las variables altura de planta y número de hojas. Para determinar área foliar, volumen de raíces y peso seco de la parte aérea de la planta se cosecharon las mismas seis plántulas de cada unidad experimental que se utilizaron para las mediciones de altura de planta y número de hojas.

Las mediciones de laboratorio se efectuaron en el Laboratorio de Suelos y Fisiología Vegetal del CIAA de La Universidad Jorge Tadeo Lozano, ubicado en Chía, Cundinamarca. El área foliar se determinó a través de un medidor de área foliar marca Conveyor Belt Unit Area Measurement System ${ }^{\circledR}$ y secando el material vegetal a $70^{\circ} \mathrm{C}$ durante $48 \mathrm{~h}$. El volumen de raíces se evaluó por el método de desplazamiento volumétrico de agua en probeta.

Para el análisis de la información se utilizó el software Statistical Análisis System, versión 9.1.3 (SAS, 1999). Se empleó análisis de varianza, utilizando el procedimiento Anova, y se aplicó 
la prueba de comparación múltiple según Tukey para todas las variables.

\section{RESULTADOS Y DISCUSIÓN}

\section{Germinación y emergencia}

En laboratorio se obtuvo $100 \%$ de germinación. No se presentó diferencia significativa $(P>0,05)$ en la velocidad de germinación entre los dos tamaños de la semilla; sin embargo, la que tendió a germinar más rápidamente y registró primero el $100 \%$ fue la de $1,1071 \mathrm{mg}$, mientras la semilla de 0,9161 mg se demoró un día más. Según Matilla (2008), la duración de las fases de germinación depende de las características de la semilla, en las cuales el tamaño, aparte del contenido de los sustratos hidratables, permeabilidad de la cubierta seminal y toma de $\mathrm{CO}_{2}$, entre otros, juega un papel importante.

La emergencia de plántulas en invernadero se presentó a partir de los 13 d después de siembra (dds) (figura 1). Según el análisis de varianza existieron diferencias significativas para esta variable con respecto a los diferentes tipos de sustrato, pues el valor correspondiente a la probabilidad de $\mathrm{F}$ fue menor de 0,05 a partir del día 15 hasta el día 38.
El sustrato que mejor emergencia presentó fue el TCP, con un promedio de 123,7 plántulas por bandeja de 128 alvéolos. Probablemente, el buen porcentaje de aire, el $\mathrm{pH}$ neutro y la alta capacidad de retención de agua de la turba canadiense con perlita (tabla 1) permitieron que la semilla germinara más fácilmente (Arias, 1989; Ansorena, 1994).

El sustrato que menor porcentaje de emergencia mostró fue el ALR (69,2 plántulas), posiblemente por su bajo porcentaje de retención de agua y baja porosidad de aire (tabla 1), propiedades físicas limitantes para la emergencia (Ansorena, 1994). Confirmando este fenómeno, la figura 1 muestra para el sustrato ALR un promedio de $54 \%$ de emergencia, mientras que los sustratos $\mathrm{TCR}, \mathrm{FCB}+\mathrm{C}, \mathrm{TAR}+\mathrm{C}$ y $\mathrm{FCB}$ alcanzaron promedios de $96,6 \%, 95,3 \%, 93,6 \%$ y $90,5 \%$, respectivamente, a los 38 dds.

\section{Altura de plántula}

A partir de 36 dds existieron diferencias significativas con respecto a los diferentes tipos de sustrato. El sustrato que mayor altura de plántula presentó fue el FCB, con 79,14 mm a los 51 d (figura 2); probablemente, las características favorables de este medio, como la

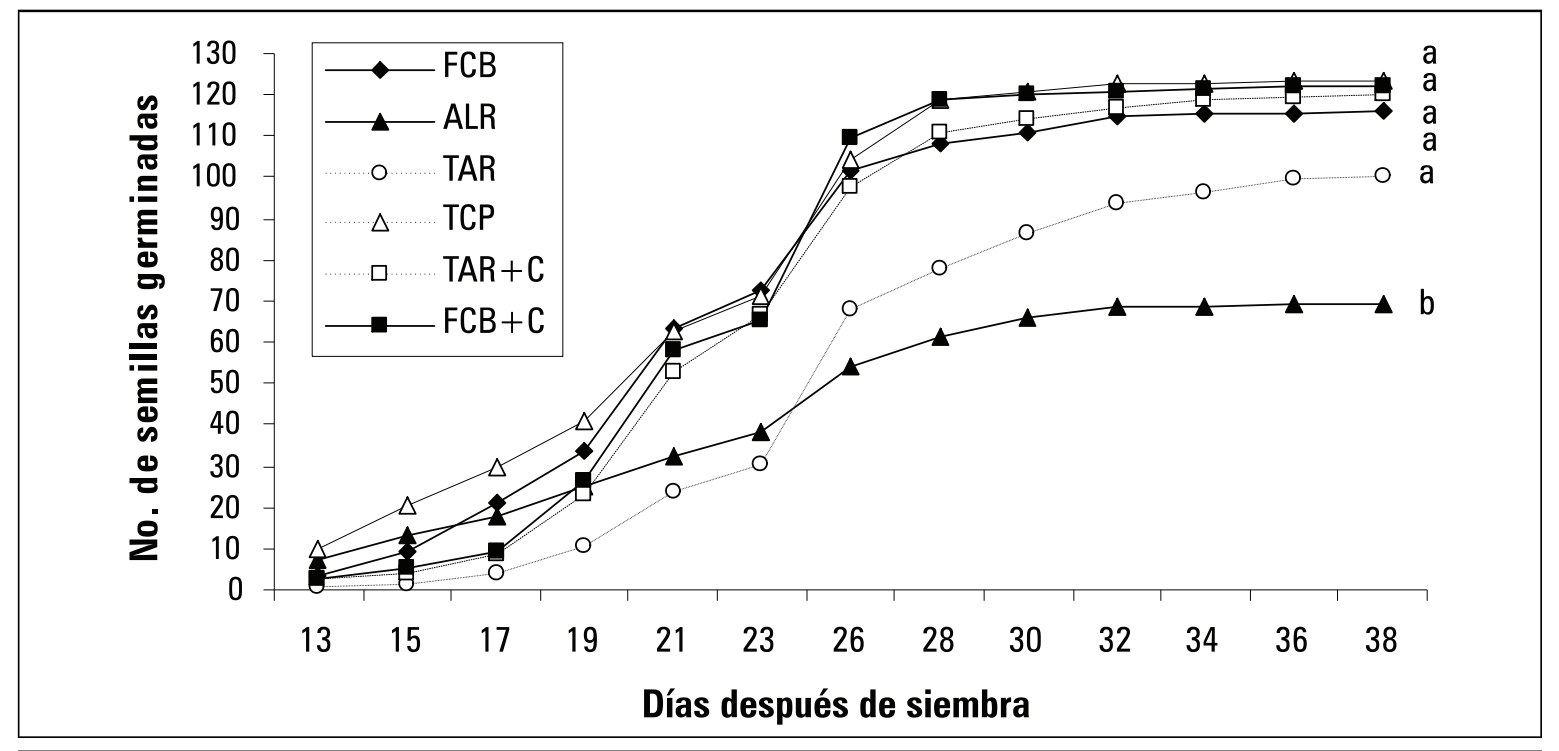

Figura 1. Número de plántulas emergidas de uchuva en invernadero entre 13 y 38 dds en bandejas de 128 alvéolos usando seis sustratos: fibra de coco brasilera (FCB); arena lavada de río fina (ALR); turba alemana rubia (TAR); turba canadiense con perlita (TCP); turba alemana rubia con cascarilla de arroz (TAR+C), y fibra de coco brasilera con cascarilla de arroz (FCB +C), hasta alcanzar 95\% de germinación. Promedios con letras distintas indican diferencia significativa a los 38 días, según la prueba de Tukey $(P<0,05)$. 
alta capacidad de humedad y retención de agua (Arias, 1989), han favorecido el crecimiento longitudinal de la planta. Así mismo, Abad et al. (2002) encontraron en 13 diferentes proveniencias de fibra de coco un alto contenido de fósforo y potasio y, además, una mayor concentración del boro, comparado con la turba, por lo cual se supone que estos nutrientes han fomentado el crecimiento longitudinal de la plántula en comparación con los otros sustratos.

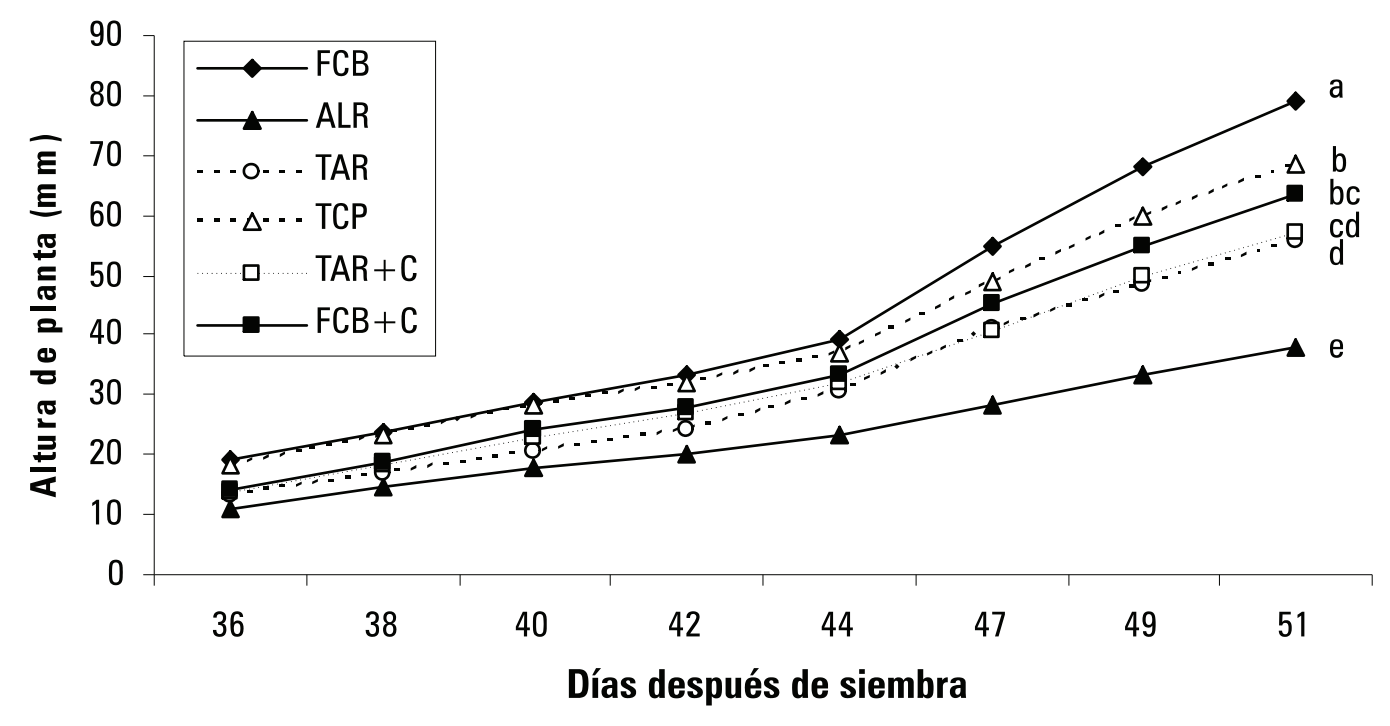

Figura 2. Crecimiento en altura de las plántulas de uchuva en los sustratos de fibra de coco brasilera (FCB), arena lavada de río fina (ALR), turba alemana rubia (TAR), turba canadiense con perlita (TCP), turba alemana rubia con cascarilla de arroz (TAR + C) y fibra de coco brasilera con cascarilla de arroz (FCB + C), luego del desarrollo del primer par de hojas verdaderas (36 dds) hasta el trasplante (51 dds). Promedios con letras distintas indican diferencia significativa a los 51 días, según la prueba de Tukey $(P<0,05)$.

El sustrato que propició la altura menor fue el ALR, con $37,86 \mathrm{~mm}$; probablemente debido a que los demás sustratos ofrecieron mejores propiedades físicas y químicas que la arena. Supuestamente, la ALR, por su baja porosidad total y volumen de aire, junto con su deficiente capacidad de retención de agua (tabla 1), ha limitado la aireación del sistema radical y la absorción de agua y nutrientes fue deficiente (Hanan, 1998). Estas características que limitan el desarrollo radical (figura 6) pueden reducir el desarrollo de la plántula (Gil y Miranda, 2007). La principal consecuencia del reducido crecimiento de la raíz es que enseguida se disminuye el crecimiento del brote (Liu y Latimer, 1995; Young et al., 1997). El bajo resultado del sustrato TAR $+C$ en el crecimiento del brote lo atribuyen Gil y Miranda (2007) a un posible contenido de taninos y residuos de plaguicidas en la cascarilla de arroz; sin embargo, esto no afectó la germinación de las semillas de uchuva (figura 1).

\section{Número de hojas}

También para esta variable existieron diferencias significativas entre los diferentes sustratos a partir de 36 dds. El crecimiento longitudinal mayor de los tallos en la fibra de coco originó un número más alto $(5,0)$ de las hojas verdaderas (en la parte vegetativa del tallo de la uchuva se inserta una hoja por nudo; Fischer, 2000), comparado con los sustratos TCP y FCP $+\mathrm{C}(4,6$ hojas) y TAR o TAR $+C$ (4,4 hojas), mientras que las uchuvas en ARL alcanzaron solamente 3,9 hojas (figura 3).

Supuestamente, el sustrato arena no suministró los nutrientes necesarios para el crecimiento del brote (Gil y Miranda, 2007), aparte de sus otras deficiencias físicas (tabla 1), lo que verifica que se debería usar solamente en combinación con otros sustratos (Hartmann et al., 2005) para los procesos de emergencia y crecimiento inicial de la plántula. Además, el alto drenaje, por la poca 


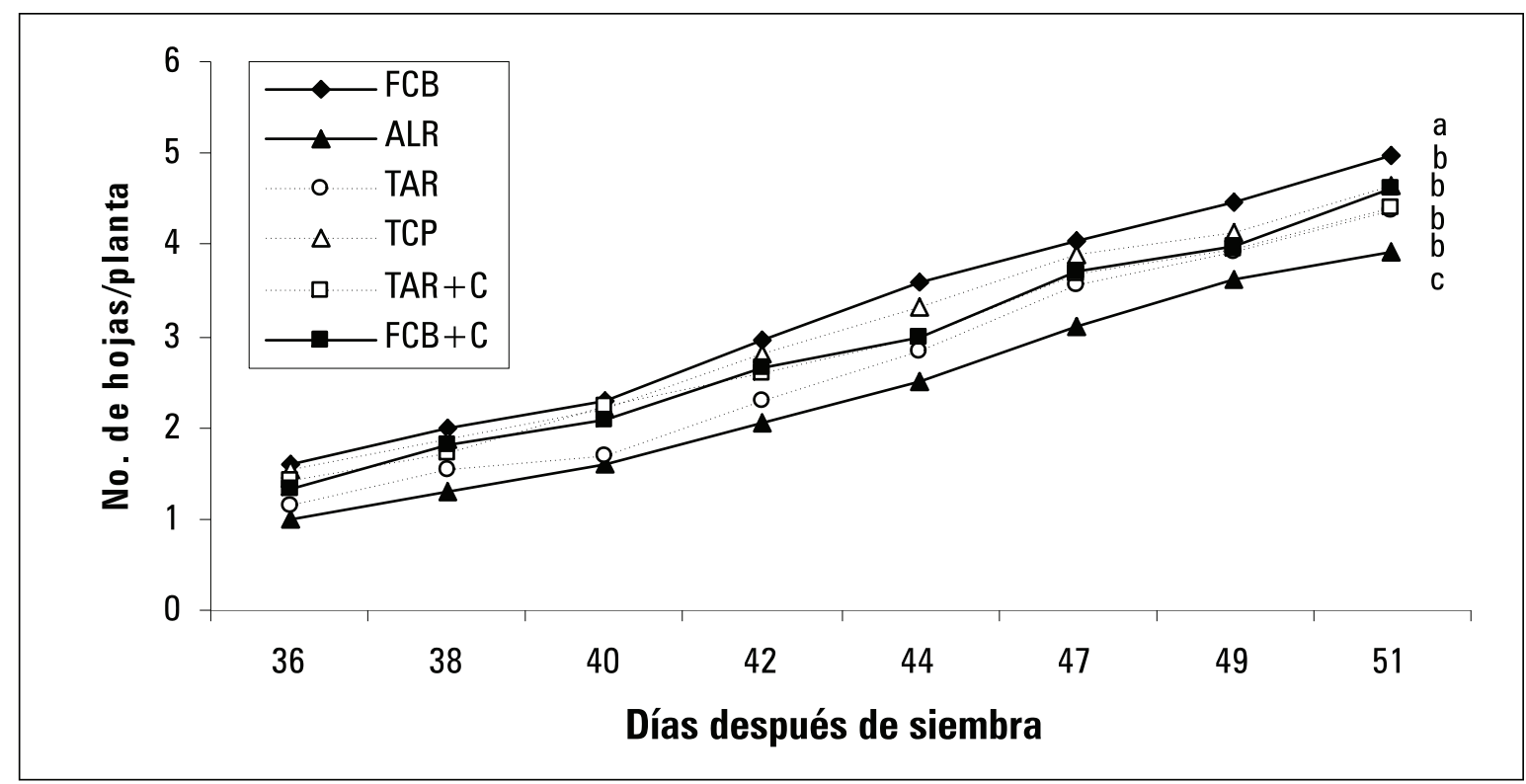

Figura 3. Desarrollo del número de hojas por planta de uchuva en los sustratos de fibra de coco brasilera (FCB), arena lavada de río fina (ALR), turba alemana rubia (TAR), turba canadiense con perlita (TCP), turba alemana rubia con cascarilla de arroz $(T A R+C)$ y fibra de coco brasilera con cascarilla de arroz $(F C B+C)$, luego del desarrollo del primer par de hojas verdaderas (36 dds) hasta el trasplante (51 dds). Promedios con letras distintas indican diferencia significativa a los 51 días, según la prueba de Tukey $(P<0,05)$.

capacidad de retención del agua, Ileva a una desecación rápida de este sustrato (Jansen et al., 1998).

\section{Área foliar}

La tabla 2 muestra que la mayor área foliar alcanzada fue en los sustratos FBC $\left(42,105 \mathrm{~cm}^{2}\right)$ y $\operatorname{TAR}(37,364)$, muy probablemente debido a que las plántulas en estos dos sustratos desarrollaron un mejor sistema radical (tabla 2), logrando mayor oportunidad para tomar más cantidad de nutrientes (Salisbury y Ross, 2000). La menor área foliar se desarrolló en el ALR $\left(14,003 \mathrm{~cm}^{2}\right)$, muy probablemente debido a que el ALR no permitió que se desarrollaran las raíces plenamente, resultando en absorción deficiente de agua y elementos minerales del sustrato (Hanan, 1998); además, el movimiento limitado de agua y solutos hacia las hojas, dentro de las membranas, impide que la presión osmótica ocurra y la demanda de elementos se reduzca (Abad et al., 2004).

Como aseguran Abad et al. (2004), la arena, por ser un sustrato inerte desde el punto de vista químico, por su capacidad casi nula de intercambio catiónico y la posible presencia de carbonatos, puede incrementar el pH del medio, lo que causa desórdenes nutricionales, afecta la toma de nutrientes y ocasiona un desarrollo deficiente del brote, dando como consecuencia un menor desarrollo foliar, como lo encontraron Gil y Miranda (2007) en plántulas de papaya creciendo en ALR.

EI desarrollo del área foliar en la TAR igualó estadísticamente el resultado favorable de la FCB, lo cual indica que la turba rubia, debido a su estabilidad estructural y su alto volumen de poros, almacena grandes cantidades de agua y aire al mismo tiempo, favoreciendo el crecimiento foliar, por lo que es la componente del sustrato más usada en la horticultura europea (Jansen et al., 1998).

\section{Masa seca de la parte aérea de la plántula}

La tabla 2 muestra que la mayor masa seca la registraron las plántulas en FCB $(0,211 \mathrm{~g})$ y TAR $(0,194 \mathrm{~g})$, probablemente debido a que las turbas rubias y en general los materiales de origen orgánico presentan un marcado efecto estimulador sobre el crecimiento y desarrollo vegetal, lo que se ha atribuido a la presencia de activadores del crecimiento tales como 


\begin{tabular}{|c|c|c|c|c|c|c|}
\hline Variable & FCB & ARL & TAR & TCP & $\mathrm{TAR}+\mathrm{C}$ & $\mathrm{FCB}+\mathrm{C}$ \\
\hline Emergencia (\%) & $90,5 \mathrm{a}$ & $54,0 \mathrm{~b}$ & $78,3 \mathrm{a}$ & 96,6 a & $93,6 \mathrm{a}$ & 95,3 a \\
\hline Área foliar $\left(\mathrm{cm}^{2}\right)$ & $42,105 \mathrm{a}$ & $14,003 \mathrm{~d}$ & $37,364 \mathrm{a}$ & $30,797 \mathrm{~b}$ & $22,631 \mathrm{c}$ & $28,435 \mathrm{cb}$ \\
\hline Masa seca parte aérea (g) & $0,211 \mathrm{a}$ & $0,091 \mathrm{~d}$ & $0,194 a b$ & $0,173 \mathrm{bc}$ & $0,144 \mathrm{c}$ & $0,157 \mathrm{c}$ \\
\hline Volumen de raíz $\left(\mathrm{cm}^{3}\right)$ & $1,733 a$ & $0,621 \mathrm{c}$ & $1,730 \mathrm{a}$ & 1,793 a & $1,633 \mathrm{a}$ & $1,280 \mathrm{~b}$ \\
\hline
\end{tabular}

compuestos de naturaleza hormonal y sustancias húmicas y fúlvicas, entre otras (Abad et al., 2004; Ansorena, 1994). La similitud en los resultados favorables de los sustratos FCB y TAR confirma que la fibra de coco puede sustituir la turba (Abad et al., 2002) en el crecimiento inicial de las plántulas; opción favorable, además, si se considera que la FCB es un material localmente disponible en los trópicos.

La producción de la menor masa seca en ALR se puede atribuir a que este sustrato no permitió que se desarrollaran las raíces plenamente (tabla 2), lo que causó una deficiente absorción de minerales en las solución del sustrato (Ansorena, 1994).

Referente al tamaño de la semilla, las de 1,107 mg originaron significativamente $(P<0,05)$ una mayor masa seca de la plántula, mientras las de 0,916 mg produjeron plántulas de menor biomasa (tabla 3). Poco después de que la semilla germina, los sistemas radical y aéreo jóvenes comienzan a utilizar los nutrientes minerales, grasas, almidón y proteínas presentes en las células de almacenamiento de la semilla (Salisbury y Ross, 2000), por lo cual se supone que las semillas más pesadas activaron más reservas que originaron una formación de biomasa seca más alta que las livianas. La semilla más grande no solo germinó más rápidamente, sino también mostró un crecimiento más vigoroso, que se expresó en una mayor acumulación de biomasa de la plántula, que Hartmann et al. (2005) destacan como características adicionales de alta calidad de germinación y que se presenta a menudo, excepto cuando se siembran semillas extremamente grandes (Jansen et al., 1998). Resultados similares encontraron Lima et al. (2005) en semillas más grandes de frijol común (Phaseolus vulgaris L.), las cuales fomentaron la biomasa de la parte aérea y subterránea de la planta y el índice de área foliar, comparado con las más livianas, especialmente en el crecimiento inicial de la planta; estos autores recomendaron el uso de semillas más pesadas para favorecer el establecimiento de cultivos en sitios con condiciones de estrés.

\section{Volumen de raíz}

El embrión de la semilla contiene la radícula o meristemo radical, del cual se desarrolla la raíz pivotante (Kozlowski y Pallardi, 1997). La formación de la raíz finaliza la germinación e inicia el crecimiento de la plántula (Matilla, 2008); es de suma importancia para su desarrollo el agua, los nutrientes minerales y los nutrimentos orgánicos; estos últimos son muy importantes al inicio del crecimiento del brote, $y$, además, son reguladores de crecimiento (citoquininas y giberelinas), sintetizados en ella (Gil, 1999).

Los sustratos TCP, FCB, TAR y TAR + C produjeron los volúmenes de raíces más altos

\begin{tabular}{|c|c|c|}
\hline \multirow{2}{*}{ Variable } & \multicolumn{2}{|c|}{ Peso de semilla } \\
\hline & $1,107 \mathrm{mg}$ & $0,916 \mathrm{mg}$ \\
\hline Altura (mm) & $61,51 \mathrm{a}$ & 59,18 a \\
\hline No. de hojas & $4,54 \mathrm{a}$ & $4,44 \mathrm{a}$ \\
\hline Área foliar $\left(\mathrm{cm}^{2}\right)$ & $29,445 \mathrm{a}$ & $29,000 \mathrm{a}$ \\
\hline Masa seca parte aérea $(\mathrm{g})$ & $0,169 a$ & $0,155 \mathrm{~b}$ \\
\hline Volumen de raíz $\left(\mathrm{cm}^{3}\right)$ & $1,495 a$ & $1,435 \mathrm{a}$ \\
\hline
\end{tabular}

Promedios con letras distintas indican diferencia significativa según la prueba de Tukey $(P<0,05)$. 
(entre 1,633 y $1,793 \mathrm{~cm}^{3}$ ), sin presentarse diferencias significativas entre ellos en esta variable (tabla 2). Las turbas en los sustratos favorecieron la retención de agua y proveyeron alta porosidad (Hanan, 1998), mientras la perlita (TCP) aumentó la aeración del sustrato, absorbiendo tres a cuatro veces su peso en agua (Hartmann et al., 2005), que se manifestó en un buen desarrollo del sistema radical.

EI $F C B+C$ y el $A L R$, con 1,280 y $0,621 \mathrm{~cm}^{3}$, produjeron el menor volumen radical, respectivamente (tabla 2). La ALR, por presentar compactación en el sustrato (Ansorena, 1994) y ser pobremente aireada (Abad et al., 2004), limitó el desarrollo radical normal.

La fibra de coco brasilera con la cascarilla de arroz $(50 \% \mathrm{c} / \mathrm{u})$ no fue una combinación favorable para el crecimiento radical, y afectó también el crecimiento de la plántula en general (figuras 23 ), supuestamente, la cascarilla, por su posible contenido de taninos y residuos de plaguicidas (Gil y Miranda, 2007), limitó mucho el desarrollo radical en mezcla con FCB, mientras este efecto no fue tan acentuado en mezcla con la turba $(T A R+C)$, que presenta cierta capacidad de amortiguamiento (Jansen et al., 1998). También en tomate, la turbia rubia, mezclada con cascarilla de arroz quemada, fomentó el crecimiento longitudinal de la raíz, comparado con la mezcla con arena o con cascarilla de arroz (no quemada), con una lámina de riego de $120 \%$ (Deaquiz-Oyola et al., 2008). La cascarilla de arroz tiene importancia en suelos pesados por su buen drenaje y la aireación de las raíces (Hanan,
1998), que no fue el caso en el presente estudio con la uchuva.

\section{CONCLUSIONES}

- El peso más alto de la semilla favorece la producción de biomasa seca de la plántula de uchuva.

- La fibra de coco brasilera generó plántulas de mayor altura y número de hojas, mientras este sustrato y la turba alemana rubia indujeron un área foliar y una masa seca de plántulas más altas.

- El volumen de raíz, indicador importante para la absorción de agua y nutrientes, fue promovido en todos los sustratos del estudio que contuvieron turba, y también en la de fibra de coco brasilera.

- El efecto favorable de la fibra de coco brasilera para el crecimiento inicial de la plántula se reduce al mezclarla con cascarilla de arroz.

- La arena lavada del río, por sus deficientes características físicas y químicas, limita el crecimiento de la plántula, especialmente por su efecto perjudicial en el desarrollo radical; además, reduce la emergencia de plántulas, en comparación con los otros sustratos del estudio.

- La fibra de coco es un material orgánico muy apto para sustituir la turba en el crecimiento inicial de las uchuvas.

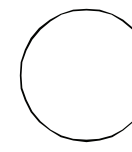

AGRADECIMIENTOS

Los autores agradecen a Juan Díaz Santanilla, por su asesoría, en la parte estadística y a Hugo Montenegro, por el suministro de las frutas de uchuva.

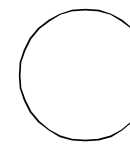

Abad, M.; P. Noguera y C. Carrión. 2004. Los sustratos en los cultivos sin suelo. pp. 113-158. En: Urrestarazu, M. (ed.). Tratado de cultivos sin suelo. Ediciones Mundi-Prensa, Madrid.

\section{REFERENCIAS BIBLIOGRÁFICAS}

Abad, M.; P. Noguera; R. Puchades; A. Maquieira y V. Noguera. 2002. Physico-chemical and chemical properties of some coconut coir dusts for use as a peat substitute for containerised ornamental plants. Bioresource Technol. 82, 241-245. 
Almanza, P. 2000. Propagación. pp. 27-40. En: Flórez, V.J.; Fischer, G. y A.D. Sora (eds.). Producción, poscosecha y exportación de la uchuva (Physalis peruviana L.). Unibiblos, Universidad Nacional de Colombia, Bogotá.

Almanza, P.J. y C.J. Espinosa. 1995. Desarrollo morfológico y análisis físico químico de frutos de uchuva Physalis peruviana L. para identificar el momento óptimo de cosecha. Trabajo final. Especialización en Frutales de Clima Frío, Facultad de Ciencias Agropecuarias, Universidad Pedagógica y Tecnológica de Colombia, Tunja.

Ansorena, M. 1994. Sustratos: Propiedades y caracterización. Ed. Mundi-Prensa, Bilbao.

Arias, R. 1989. Caracterización de las propiedades físicas de 10 materiales, descripción, su uso potencial como sustratos y evaluación del crecimiento de plántulas de lechuga (Lactuca sativa L.) en los materiales promisorios como sustratos. Trabajo de grado. Facultad de Agronomía, Universidad Nacional de Colombia, Bogotá.

Borscak, J.D. y F. Covatta. 2007. Propagación sexual de especies frutales. pp. 105-131. En: Sozzi, G.O. (ed.). Árboles frutales. Ecofisiología, cultivo y aprovechamiento. Editorial Facultad de Agronomía, Universidad de Buenos Aires, Buenos Aires.

Brücher, H. 1977. Tropische Nutzpflanzen. SpringerVerlag, Berlin. pp. 394-395.

Celis-Velázquez, R.; C.B. Peña-Valdivia; M. LunaCavazos y R. Aguirre. 2005. Caracterización morfológica de las semillas y consumo de reservas durante la emergencia de plántulas de frijol (Phaseolus vulgaris L.) silvestre y domesticado. Rev. Fac. Agron. (LUZ) 27(1), 61-87.

Deaquiz-Oyola, Y.; J. Álvarez-Herrera y A. Fraile. 2008. Efecto de diferentes láminas de riego y sustratos en la propagación de tomate (Solanum lycopersicum L.). Rev. Colomb. Cienc. Hortíc. 2(1), 54-65.

Fischer, G. 2000. Crecimiento y desarrollo. pp. 9-26. En: Flórez, V.J.; G. Fischer y A.D. Sora (eds.): Producción, poscosecha y exportación de la uchuva (Physalis peruviana L.). Unibiblos, Universidad Nacional de Colombia, Bogotá.

Fischer, G.; G. Ebert y P. Lüdders. 2007. Production, seeds and carbohydrate contents of cape gooseberry (Physalis peruviana L.) fruits grown at two contrasting Colombian altitudes. J. Appl. Bot. Food Qual. 81, 29-35.

Gil, A. y D. Miranda. 2007. Efecto de cinco sustratos sobre índices de crecimiento de plantas de papaya (Carica papaya L.) bajo invernadero. Rev. Colomb. Cienc. Hortíc. 1(2), 142-253.
Gil, G.F. 1999. Fruticultura: El potencial productivo. Alfaomega Grupo Editor, México D. F.

Hanan, J.J. 1998. Greenhouses. Advanced technology for protected horticulture. CRC Press, Boca Raton, FL. pp. 307-313.

Hartmann, H.T.; D. E. Kester; F.T. Davies y R.L. Geneve. 2005. Plant propagation: principles and practices. $7^{\text {th }}$ ed.. Prentice Hall, Upper Saddle River, NY.

Kozlowski. T.T. y S.G. Pallardy. 1997. Physiology of woody plants. $2^{\text {nd }}$ ed. Academic Press, San Diego, CA.

Jansen, H; E. Bachthaler; E. Fölster y H.-C. Scharpf. 1998. Gärtnerischer Pflanzenbau. Verlag UImer, Stuttgart, Alemania.

Legge, A. P. 1974. Notes on the history, cultivation and uses of Physalis peruviana L. J. Royal Hort. Soc. 99(7), 310-314.

Lima, E.R.; A.S. Santiago; A.P. Araújo y M.G. Teixeira. 2005. Effects of seed size of sown seed on growth and yield of common bean cultivars of different seed sizes. Braz. J. Plant Physiol. 17(3), 273-281.

Liu, A. y J. G. Latimer. 1995. Water relations and abscisic acid levels of watermelon as affected by rooting volume restriction. J. Exp. Bot. 46, 1011-1015.

Matilla, A.J. 2008. Desarrollo y germinación de las semillas. pp. 537-558. En: Azcón-Bieto, J. y M. Talón (eds.). Fundamentos de fisiología vegetal. 2da ed. McGraw-Hill Interamericana, Madrid.

Peña, J.F.; J.D. Ayala; G. Fischer; B. Chaves; J.F. Cárdenas y P.J. Almanza. 2010. Relaciones semilla fruto en tres ecotipos de uchuva (Physalis peruviana L.). Rev. Colomb. Cienc. Hortic. 4(1), 43-54.

Ryugo, K. 1993. Fruticultura: Ciencia y arte. AGT Editor, México D.F.

SAS. 1999. SAS/STAT User's Guide. Versión 8. Vol. 2. SAS Institute, Cary, NC.

Salisbury, F.B. y C.W. Ross. 2000. Fisiología de las plantas. Editorial Paraninfo Thomson Learning, Madrid.

Schopfer, P. y A. Brennicke. 2006. Pflanzenphysiologie. Elsevier $\mathrm{GmbH}$, Munich, Alemania.

Valencia, M.L. 1985. Anatomía del fruto de la uchuva. Acta Biol. Colomb. 1(2), 63-89.

Whitson, M. y P.S. Manos. 2005. Untangling Physalis (Solanaceae) from the Physaloids: A two-gene ohykigeny of the Physalinae. Syst. Bot. 30(1), 216230.

Young, I.M.; K. Montagu y J.A.G. Conroy. 1997. Mechanical impedance of root growth directly reduces leaf elongation rates of cereals. New Phytologist 135, 613-619. 